\title{
Expression of the soluble vascular endothelial growth factor receptor-1 in cutaneous melanoma: role in tumour progression
}

\author{
F. Ruffini, C.M. Failla, ${ }^{\star}$ A. Orecchia, ${ }^{\star}$ M.R. Bani, $\uparrow$ A.S. Dorio, $\$$ C. Fortes, $\S$ G. Zambruno, ${ }^{\star}$ G. Graziani, $\$$ \\ R. Giavazzi, $\dagger$ S. D’Atri and P.M. Lacal \\ Laboratory of Molecular Oncology, *Laboratory of Molecular and Cell Biology and §Clinical Epidemiology Unit, Istituto Dermopatico dell'Immacolata-IRCCS, \\ 00167 Rome, Italy \\ †Laboratory of the Biology and Treatment of Metastasis, Department of Oncology, Mario Negri Institute for Pharmacological Research, Milan, Italy \\ $\ddagger$ Department of Neuroscience, University of Rome 'Tor Vergata', Rome, Italy
}

\section{Summary}

Correspondence

Pedro M. Lacal.

E-mail: p.lacal@idi.it

\section{Accepted for publication}

21 December 2010

\section{Funding sources}

This study was supported by the Italian Ministry of Health (RF07 Onc. 25) and by a FIRB grant (RBIP061.CA9).

Conflicts of interest

None declared.

DOI $10.1111 / j .1365-2133.2010 .10200 . x$
Background Vascular endothelial growth factor (VEGF)-A, placenta growth factor (PlGF) and their corresponding membrane receptors are involved in autocrine and paracrine regulation of melanoma growth and metastasis. Besides the membrane receptors, a soluble form of the VEGF receptor (VEGFR)-1 (sVEGFR-1) has been identified, that behaves both as a decoy receptor, sequestering VEGF-A and PlGF, and as an extracellular matrix (ECM) molecule, promoting endothelial cell adhesion and migration through the interaction with $\alpha 5 \beta 1$ integrin.

Objectives To analyse whether sVEGFR-1 plays a role during melanoma progression.

Methods sVEGFR-1 expression was evaluated in a panel of 36 melanoma cell lines and 11 primary human melanocyte cultures by quantitative real-time polymerase chain reaction analysis and in specimens of primary or metastatic melanoma lesions from 23 patients by immunohistochemical analysis.

Results sVEGFR-1 expression was highly upregulated in melanoma cell lines with respect to human melanocytes. Interestingly, cell lines obtained from cutaneous metastases showed a significant reduction of sVEGFR-1 expression, as compared with cell lines derived from primary tumours. These results were confirmed by immunohistochemical analysis of sections from primary skin melanomas and the corresponding cutaneous metastases, suggesting that modulation of sVEGFR-1 expression influences ECM invasion by melanoma cells and metastasis localization. Moreover, we provide evidence that adhesion of melanoma cells to sVEGFR-1 is favoured by the activation of a VEGF-A/VEGFR-2 autocrine loop. Conclusions Our data strongly suggest that sVEGFR-1 plays a role in melanoma progression and that low sVEGFR-1/VEGF-A and sVEGFR-1/transmembrane VEGFR-1 ratios might predict a poor outcome in patients with melanoma.
A critical step in melanoma progression is the transition of the primary tumour from a radial growth phase (RGP) to a vertical growth phase (VGP). The latter requires high angiogenic activity, that contributes to the metastatic dissemination of tumour cells. Melanoma cells release several angiogenic factors, including the members of the vascular endothelial growth factor (VEGF) family, VEGF-A and placenta growth factor (PlGF). ${ }^{1-3}$ These growth factors are involved in the autocrine and paracrine regulation of melanoma progression and, in particular, VEGF-A expression has been correlated with the transition of melanoma lesions from the RGP to the VGP. ${ }^{4,5}$ Melanoma cells express functional receptors for these angiogenic factors, and the receptor/ligand interaction modulates cellular pathways important for melanoma cell proliferation, apoptosis and invasiveness. . $2,6-8^{-8}$

VEGF-A exerts its biological effects on endothelial cells through the interaction with two membrane tyrosine kinase receptors, VEGF receptor (VEGFR)-1 and VEGFR-2. ${ }^{9}$ VEGFR-1 
mainly mediates endothelial cell migration in response to the angiogenic factor, while VEGFR-2 is the major receptor involved in endothelial cell survival and proliferation and in the increase of microvascular permeability. PlGF binds exclusively to VEGFR-1 and promotes endothelial cell migration. ${ }^{9}$

In addition to the transmembrane form of VEGFR-1 (mVEGFR-1), endothelial cells produce a soluble form of the receptor (sVEGFR-1), deriving from an alternative splicing of the same gene transcript. ${ }^{10}$ sVEGFR-1, which comprises the extracellular region of mVEGFR-1 and a specific sequence of 30 amino acids at the C-terminal domain, can prevent the interaction of VEGF-A and PlGF with the tyrosine kinase transmembrane receptors. ${ }^{11}$ It has been demonstrated that sVEGFR-1 is sufficient to rescue vessel branching defects occurring during vasculogenesis in mVEGFR-1 knockout mice. ${ }^{12}$ In agreement with this finding, studies by our group demonstrated that sVEGFR-1 is a component of the extracellular matrix (ECM) and that it interacts directly with the $\alpha 5 \beta 1$ integrin, leading to endothelial cell adhesion and migration. ${ }^{13}$ Therefore, sVEGFR-1 could play distinct roles during angiogenesis, as a decoy receptor, and as a molecule involved in cell adhesion and migration.

We previously described the expression of mVEGFR-1 in melanoma cell lines, and suggested a role for this receptor in melanoma growth and invasiveness. ${ }^{2}$ However, no data are available so far on the expression of sVEGFR-1 in melanoma, and on its possible role during melanoma progression. In the present study, we analysed sVEGFR-1 expression in a panel of melanoma cell lines and specimens from primary and metastatic lesions. Moreover, with the purpose of evaluating whether sVEGFR-1 might be involved in melanoma cell invasion of the ECM, we analysed the ability of melanoma cells to adhere to sVEGFR-1. The results indicated a relationship between sVEGFR-1 expression and human melanoma progression and suggested that a low expression of sVEGFR-1, specifically occurring in cutaneous metastases with respect to primary melanomas or lymph node metastases, might contribute to the ability of melanoma to invade the skin.

\section{Materials and methods}

\section{Materials}

Culture media and supplements, Freund adjuvant for immunizations and reagents for hybridoma production, antibody purification and cell transfection, as well as antibodies for immunocytochemistry were purchased from Sigma (St Louis, MO, U.S.A.). Fatty acid-free bovine serum albumin was from Roche (Mannheim, Germany). VEGF-A, PlGF and antibodies used in enzyme-linked immunosorbent assays (ELISAs) were from R\&D Systems (Abingdon, U.K.).

\section{Melanoma cell lines and normal human cells}

The origin of the human melanoma cell lines GR-Mel, ST-Mel, SN-Mel, CN-Mel, M14, 13443-Mel, 397-Mel, WM115,
WM266-4, LCP-Mel, LCM-Mel, LB-24 and SK-Mel-28 has been described previously. ${ }^{2}$ CT-Mel, DR-Mel, CR-Mel, MR-Mel, CH-Mel, CL-Mel and DT-Mel cell lines were established in the Laboratory of Molecular Oncology, IDI-IRCCS (Rome, Italy). A375 cell line was purchased from the American Type Culture Collection (Manassas, VA, U.S.A.), M20 cell line was a generous gift from Dr G. Zupi (Regina Elena Cancer Institute, Rome, Italy) and INT021 cell line was a generous gift from Dr L. Rivoltini (National Cancer Institute, Milan, Italy). The remaining melanoma cell lines used in this study were kindly provided by Dr Meenhard Herlyn (The Wistar Institute, Philadelphia, PA, U.S.A.). Normal human melanocytes, human umbilical vein endothelial cells (HUVEC), human keratinocytes and the murine NIH/3T3 cell line, used as controls, were obtained as previously described. ${ }^{2}$

\section{Quantitative real-time polymerase chain reaction}

Quantitative real-time reverse transcription-polymerase chain reaction (qRT-PCR) was performed by the dual-labelled fluorigenic probe method, using an ABI Prism 7000 sequence detector (PerkinElmer, Groningen, the Netherlands), and expression levels were calculated by the relative standard curve method. Primers used for qRT-PCR analysis were as follows: sVEGFR-1, forward 5'-CGACTTGGTGCACGTTTGG-3' and reverse 5'-CACAGAGAAGGCAGTGCAGG-3'; mVEGFR-1, forward 5'-ACCGAATGCCACCTCCATG- ${ }^{\prime}$ and reverse 5'-AGGCCTTGGGTTTGCTGTC-3'; VEGF-A, forward 5'-GCTACTGCCATCCAATCGAG-3' and reverse $5^{\prime}$-TGGTGATGTTGGACTCCTCA-3'; VEGFR-2, forward $5^{\prime}$-GTCTATGCCATTCCTCCCCC-3' and reverse 5'-GAGACAGCTTGGCTGGGCT. ${ }^{14}$

\section{Production of mouse anti-soluble vascular endothelial growth factor receptor-1 monoclonal antibodies}

Anti-sVEGFR-1 monoclonal antibodies (mAbs) were generated by the standard hybridoma technology using BALB/C mice (Charles River, Calco, Italy) immunized with $50 \mu \mathrm{g}$ of a MAP4 peptide (Multiple Antigenic Peptide; PRIMM, Milan, Italy), which corresponds to part of the human sVEGFR-1 unique sequence (NKKAVFSRISKFKSTRND). Hybridomas producing anti-human sVEGFR-1 mAbs were identified by ELISA using sVEGFR-1-coated 96-well plates. The IgM mAb produced by the hybridoma clone H9E6 was purified from the culture supernatant by standard protocols.

\section{Flow cytometry analysis}

Evaluation of integrin $\alpha 5 \beta 1$ expression was performed using the mAb JBS5 (Chemicon, Temecula, CA, U.S.A.), as previously described. ${ }^{15}$

\section{Immunohistochemical analysis}

Twenty-three patients with melanoma, diagnosed between 1996 and 2001, were identified retrospectively from the 
Melanoma Registry, Epidemiology Unit, IDI-IRCCS Hospital, Rome. Fourteen of the 23 patients with melanoma developed cutaneous metastases between 1998 and 2005. Four-micrometre thick sections of melanoma specimens or normal skin were deparaffinized and rehydrated. Staining procedure was performed using the TSA Biotin System (PerkinElmer), following the manufacturer's instructions. Negative controls were obtained by omitting the primary antibody or by absorbing the primary antibody with 10 times molar excess of the MAP4 peptide.

\section{Generation of M14C2 cells expressing vascular endothelial growth factor receptor-2 or soluble vascular endothelial growth factor receptor-1}

The M14 cell line, devoid of both VEGFR-1 and VEGFR-2, was cloned by limiting dilution and one clone (M14C2) was transfected with the pcDNA3/VEGFR-2 plasmid (a generous gift of Dr K. Ballmer-Hofer, PSI, Zurich) or with the empty vector (pcDNA3). Transfection was performed using polybrene and dimethyl sulphoxide, as previously described, ${ }^{7}$ and transfected cells were selected in G418-containing culture medium. VEGFR-2-expressing subclones were identified by Western blot analysis using the C-20 polyclonal antibody from Santa Cruz Biotechnology (Santa Cruz, CA, U.S.A.) and HUVEC as positive control.

The sVEGFR-1 cDNA was obtained from HUVEC RNA by qRT-PCR amplification and cloned into the pBLAS49.2 plasmid (InvivoGen, San Diego, CA, U.S.A.). M14C2 cells were then transfected with the pBLAS49.2/sVEGFR-1 plasmid (M14C2/SF15 cells) or with the empty vector (M14C2/B4 cells) as described above. sVEGFR-1 expression in M14C2/SF15 cells was confirmed by Western blot analysis using the polyclonal antibody AF321 (R\&D Systems), whereas the amount of polypeptide secreted into the culture supernatant was quantified by ELISA.

\section{Results}

\section{Expression of soluble vascular endothelial growth factor receptor-1 in human melanoma cell lines}

Expression of sVEGFR-1 was initially analysed by qRT-PCR in a small group of melanoma cell lines derived from primary or metastatic lesions and in three melanocyte cultures (Fig. 1). All the melanocyte cultures were negative for sVEGFR-1 mRNA, whereas this transcript was highly expressed in three of four primary melanomas (Fig. 1). On the other hand, among the eight melanoma cell lines originating from metastases, only two cell lines displayed high levels of sVEGFR-1 mRNA (Fig. 1). The release of sVEGFR-1 into the cell culture supernatant was analysed by ELISA. As illustrated in Table 1, a good correspondence was found between the expression of sVEGFR-1 mRNA and the amount of protein secreted by the melanoma cell lines.

With the aim of identifying the subset of melanoma cell lines that preferentially expresses sVEGFR-1, the mRNA levels for this VEGFR-1 isoform were evaluated by qRT-PCR in a panel of 36 melanoma cell lines, originating from primary or metastatic lesions (Fig. 2). The expression of mVEGFR-1 was also determined in the same panel of cell lines. The results indicated that 11 of 12 (92\%) cell lines originating from primary tumours and 16 of $24(67 \%)$ cell lines derived from metastatic lesions expressed sVEGFR-1 (Fig. 2). When the metastatic cell lines derived from cutaneous or lymph node metastases were analysed separately, seven of 13 (54\%) cell lines established from skin metastases and nine of 11 cell lines $(82 \%)$ established from lymph node metastases were found to be positive for sVEGFR-1 expression. The mVEGFR-1 transcript was highly expressed in both primary (11 of 12 cell lines, 92\%) and metastatic (19 of 24 cell lines, 79\%) cell lines. In particular, $69 \%$ of the cutaneous metastases (nine of 13) and $91 \%$ of the lymph node metastases (10 of 11 ) were positive for mVEGFR-1 (Fig. 2). Primary human melanocyte cultures

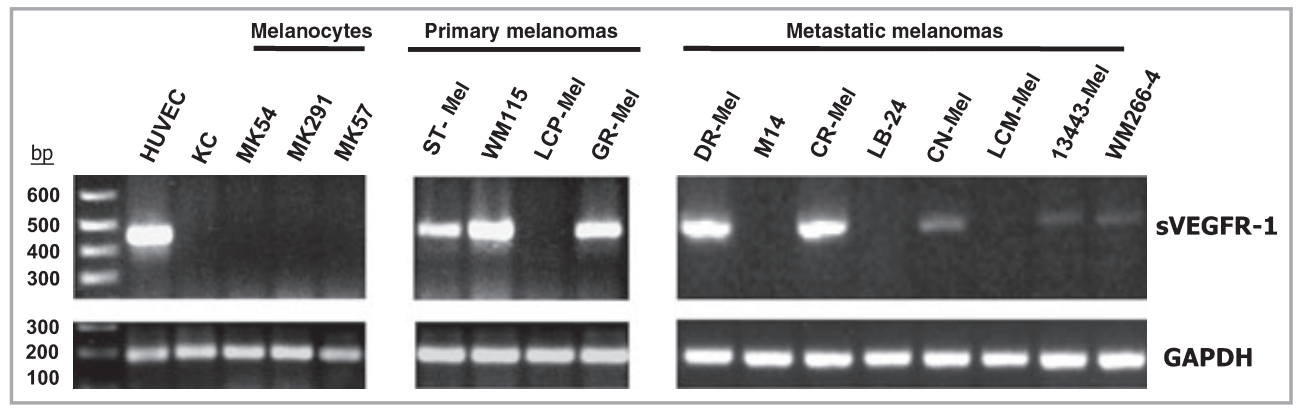

Fig 1. Expression of soluble vascular endothelial growth factor receptor-1 (sVEGFR-1) in human melanocytes and melanoma cell lines. Quantitative real-time reverse transcription-polymerase chain reaction for sVEGFR-1 and glyceraldehyde-3-phosphate dehydrogenase (GAPDH), to confirm integrity of RNA samples, was performed as previously described, ${ }^{2}$ utilizing the following primers and conditions: forward primer 5'-TCAGATCTCGGTTCTATGCTCAGCTACTGGGACACC-3', reverse primer 5'-TGGAGATCCGAGAGAAAACA-3', annealing for $30 \mathrm{~s}$ at $58{ }^{\circ} \mathrm{C}$. Reaction products were separated on $1 \%$ agarose gels. Human umbilical vein endothelial cells (HUVEC) and keratinocytes (KC) RNAs were used as positive and negative controls, respectively. Results are representative of one of three different experiments giving comparable results. 
Table 1 Soluble vascular endothelial growth factor receptor-1 (sVEGFR-1) secretion by melanoma cell lines from different origin

\begin{tabular}{ll}
\hline Cell line & $\begin{array}{l}\text { sVEGFR-1 } \\
\left(\text { ng per } 10^{6} \text { cells }\right)^{\mathrm{a}}\end{array}$ \\
\hline Primary melanomas & \\
$\quad$ GR-Mel & $3 \cdot 79 \pm 1 \cdot 11$ \\
WM115 & $2 \cdot 60 \pm 1 \cdot 08$ \\
Cutaneous metastases & \\
M14 & ND \\
WM266-4 & $0 \cdot 28 \pm 0 \cdot 03$ \\
DR-Mel & $5 \cdot 21 \pm 1 \cdot 16$ \\
LB-24 & $0 \cdot 08 \pm 0 \cdot 12$ \\
Lymph node metastases & \\
CR-Mel & $5 \cdot 64 \pm 1 \cdot 67$ \\
CN-Mel & $0 \cdot 23 \pm 0 \cdot 15$ \\
13443-Mel & $0 \cdot 19 \pm 0 \cdot 10$ \\
LCM-Mel & $0 \cdot 02 \pm 0 \cdot 03$ \\
\hline
\end{tabular}

${ }^{a}$ The amount of sVEGFR-1 released in the culture supernatant of semiconfluent cell cultures, collected as previously described, ${ }^{2}$ was evaluated utilizing an enzyme-linked immunosorbent assay kit from R\&D Systems (Abingdon, U.K.). Each value represents the mean \pm SD of three independent determinations. As a reference, the amount of sVEGFR-1 released by human umbilical vein endothelial cells under the same experimental conditions was $1.94 \pm 0 \cdot 10 \mathrm{ng}$ per $10^{6}$ cells. ND, not detectable.

were mostly negative for the expression of both MVEGFR-1 and sVEGFR-1 (Fig. 2).

Box and whisker diagram analysis of the qRT-PCR results further evidenced that the expression of both VEGFR-1 isoforms was significantly upregulated $(P<0.05)$ in melanoma cells with respect to melanocytes (Fig. 3). Moreover, the cell lines established from primary tumours displayed significantly higher $(\mathrm{P}<0.05)$ sVEGFR-1 and mVEGFR-1 mRNA levels than those of the cell lines derived from cutaneous metastases. No significant differences in the expression of sVEGFR-1 and mVEGFR-1 were, in contrast, observed between cell lines originating from primary tumours and lymph node metastases (Fig. 3a, b). The ratio between the expression of sVEGFR-1 and mVEGFR-1 mRNAs in each cell line was evaluated. Interestingly, the mean ratio in the cutaneous metastasis cell lines almost halved with respect to the primary melanomas (Fig. 3c).

The analysis of VEGF-A mRNA showed that primary and cutaneous metastases expressed comparably high levels of this transcript (Fig. 3d).

\section{Soluble vascular endothelial growth factor receptor-1 expression in human melanoma specimens}

To verify whether the expression of sVEGFR-1 observed in melanoma cell lines was representative of the in vivo situation and was not related to culture conditions, we then analysed primary and metastatic human melanoma specimens by immunohistochemistry using the anti-sVEGFR-1
mAb H9E6, generated in our laboratories. Specificity of H9E6 mAb for sVEGFR-1 was initially verified by ELISA as described in Materials and methods and then confirmed by immunofluorescence using a melanoma cell line (M14C2/SF15) expressing only sVEGFR-1. This cell line was obtained by transfection of a plasmid encoding sVEGFR-1 into a clone of M14 cells devoid of both VEGFR-1 and VEGFR-2 (clone M14C2). As shown in Figure 4, H9E6 mAb stained M14C2/SF15 cells, whereas it did not react with cells transfected with the empty vector (subclone M14C2/B4).

Twenty-three specimens obtained from patients with primary cutaneous melanoma, with Breslow thickness ranging from 0.8 to $9.0 \mathrm{~mm}$, were then analysed for sVEGFR-1 expression. Fourteen cutaneous metastases derived from the same patients were also available. Cytoplasmic expression of sVEGFR-1 was observed in 22 of the 23 primary melanomas, although the percentage of positive tumour cells varied among the samples (Fig. 5, Table 2). Signal specificity of H9E6 was indicated by the abrogation of section staining after preincubation of the mAb with a 10-fold molar excess of the peptide used as immunogen for its production (results not shown). Notably, 10 of the 14 cutaneous metastases showed a lower level of sVEGFR-1 expression when compared with the corresponding primary melanoma (Table 2). The expression of sVEGFR-1 was also analysed in normal human skin and, consistently with previous findings, ${ }^{16,17}$ dermal endothelial cells and keratinocytes of the basal epidermal layer stained weakly with the anti-sVEGFR-1 mAb (Fig. 5).

\section{Melanoma cell adhesion to soluble vascular endothelial growth factor receptor-1}

We previously demonstrated that SVEGFR-1 secreted by endothelial cells is deposited into the ECM, and that sVEGFR-1 immobilized on a solid support can directly interact with the $\alpha 5 \beta 1$ integrin expressed on endothelial cells favouring cell adhesion. ${ }^{13}$ Therefore, we investigated whether melanoma cells might also be able to adhere to sVEGFR-1 using cell lines expressing high levels of $\alpha 5 \beta 1$ integrin (85-100\% positive cells, as evaluated by flow cytometry analysis, data not shown). The results indicated that only four of nine cell lines tested adhered to sVEGFR-1 (Table 3).

As we previously demonstrated that activation of a VEGF-A/ VEGFR-2 autocrine loop promotes melanoma cell invasiveness, ${ }^{7}$ we analysed the secretion of VEGF-A and the expression of VEGFR-2 by the cell lines under investigation (Table 3). Interestingly, we observed that a high sVEGFR-1 expression was associated with undetectable amounts of VEGF-A in the culture supernatants of melanoma cells (GR-Mel, WM115, DR-Mel and CR-Mel). Moreover, these cell lines and also the cell line that does not express VEGFR-2 (M14) were not able to adhere to sVEGFR-1-coated surfaces (Table 3). Therefore, we hypothesized that the simultaneous expression of VEGF-A and VEGFR-2 might be important for the adhesion of melanoma cells to sVEGFR-1. 


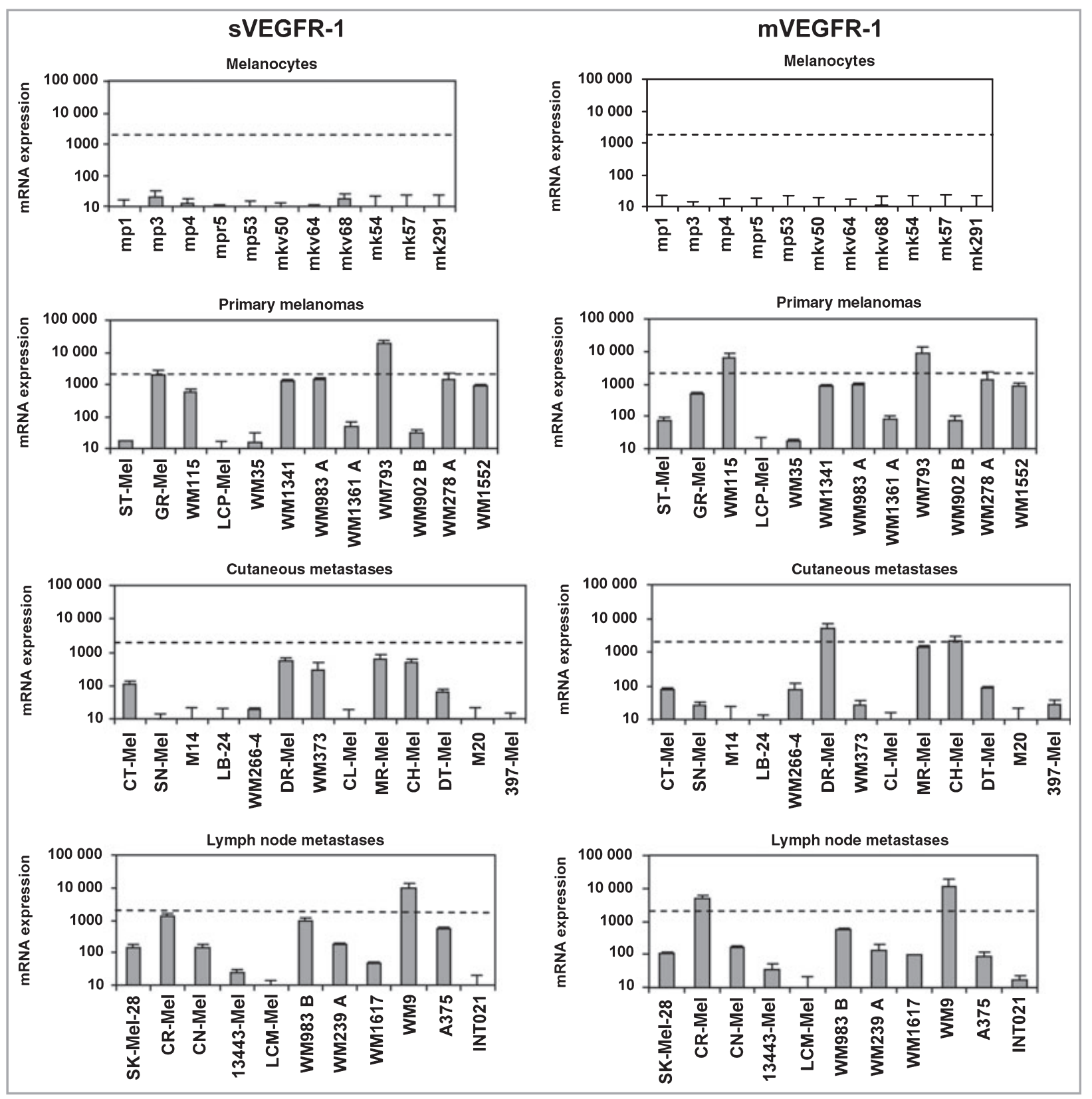

Fig 2. Expression of soluble vascular endothelial growth factor receptor-1 (sVEGFR-1) and transmembrane vascular endothelial growth factor receptor-1 (mVEGFR-1) in human melanocytes and melanoma cell lines. One microgram of total RNA was reverse transcribed in $20 \mu \mathrm{L}$ reaction containing Random Primers and Superscript II enzyme (Invitrogen, Paisley, U.K.). For each sample, the level of sVEGFR-1 and mVEGFR-1 transcripts was normalized to that of $18 \mathrm{~S}$ RNA and referred to the values of the mVEGFR-1- and sVEGFR-1-negative M14 cell line, to which the arbitrary value of 1.0 was assigned. Each histogram represents the mean \pm SD value of at least three independent determinations. Dotted lines indicate the expression value obtained for human umbilical vein endothelial cells in the same assay (i.e. $2373 \pm 336$ for mVEGFR-1, and $2484 \pm 785$ for sVEGFR-1).

To verify our hypothesis, the M14C2 clone, devoid of both VEGFR-1 and VEGFR-2, was stably transfected with a plasmid containing the VEGFR-2 cDNA (M14C2/MK18 subclone) or with the empty vector (M14C2/C4 subclone) (Fig. 6a). The transfected subclones, both secreting VEGF-A (data not shown), were then tested for their ability to adhere to sVEGFR-1. The results indicated that
M14C2/MK18 cells adhered three times more efficiently to this polypeptide than $\mathrm{M} 14 \mathrm{C} 2 / \mathrm{C} 4$ cells (Fig. 6b). The improved adhesion of M14C2/MK18 cells was not due to different levels of $\alpha 5 \beta 1$ integrin expression, as both subclones adhered to a similar extent to fibronectin, the ECM component to which the activated $\alpha 5 \beta 1$ integrin preferentially binds (Fig. 6b). As shown in Figure 6b, the adhesion 

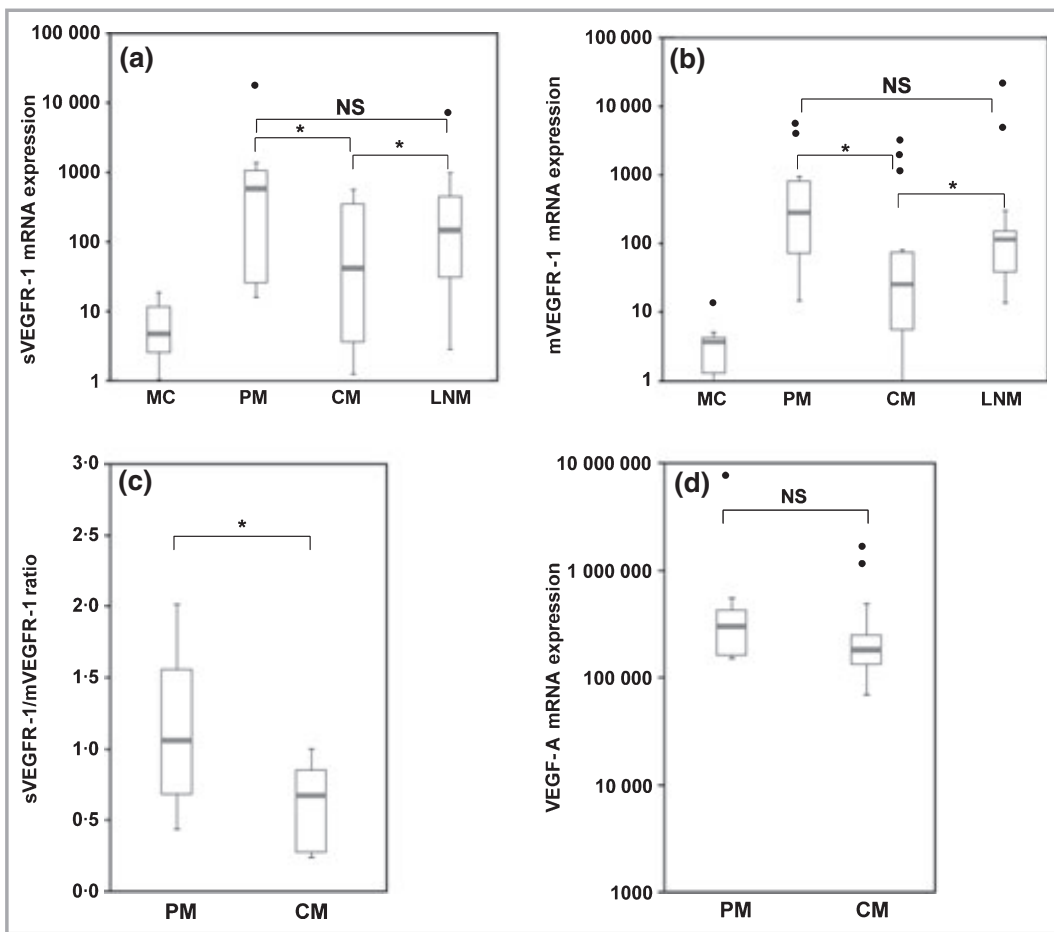

Fig 3. Box and whisker plots for the relative soluble vascular endothelial growth factor (VEGF) receptor-1 (sVEGFR-1), transmembrane VEGF receptor-1 (mVEGFR-1) mRNA and VEGF-A expression in human melanocytes and melanoma cells. The top and bottom of each box represent the 75th and 25th percentile, respectively, and whiskers the 10th and 90th percentiles. The horizontal bar within each box indicates the median and dots are outliers (values beyond 1.5 times the interquartile range). MC, melanocytes; PM, primary melanomas; CM, cutaneous metastases; LNM, lymph node metastases. Data were analysed by ANova for multiple comparisons followed by Bonferroni test. The asterisk (*) indicates statistically significant differences $(\mathrm{P}<0.05)$. NS, not significant. $(\mathrm{a}, \mathrm{b})$ Box and whisker plots for sVEGFR-1 and mVEGFR-1 expression in human MC, PM, $\mathrm{CM}$ and LNM, using data presented in Figure 2. The differences between MC and all the other groups were always statistically significant $(P<0 \cdot 05)$. (c) Data from Figure 2 were used to calculate the ratio of sVEGFR-1 and mVEGFR-1 mRNA expression in each cell line derived from PM and CM. (d) Expression of VEGF-A mRNA in the melanoma cell lines originating from PM or CM. VEGF-A expression was normalized for each sample as described in the legend to Figure 2, except that the arbitrary value of 1.0 was assigned to the NIH/3T3 cell line, which was used as negative control.

to gelatin-coated wells did not differ between VEGFR-2 positive and negative cells.

Adhesion of M14C2/MK18 cells to sVEGFR-1 was significantly downmodulated by anti-VEGF-A or anti-VEGFR-2 neutralizing antibodies (Fig. 6c), further supporting the involvement of the VEGF-A/VEGFR-2 signalling pathway in the modulation of melanoma cell adhesion to sVEGFR-1.

\section{Discussion}

Previous investigations by our group have demonstrated the secretion of VEGF-A and PlGF and the expression of their membrane tyrosine kinase receptors VEGFR-1 and VEGFR-2 in a large number of melanoma cell lines. ${ }^{2}$ Moreover, we and others have suggested a role for these growth factors and receptors in melanoma growth and progression. ${ }^{1,2,6-8}$ However, no data are presently available on the expression of sVEGFR-1 in melanoma and on its possible role in the onset and/or progression of this malignancy.

In the present study we show, for the first time, that the expression of sVEGFR-1 is markedly upregulated in melanoma cell lines with respect to normal melanocytes. In particular, most melanoma cell lines derived from primary tumours or lymph node metastases display high levels of sVEGFR-1 mRNA, while the transcripts for this VEGFR-1 isoform are absent or barely detectable in primary cultures of normal melanocytes. Also in cell lines established from cutaneous metastases sVEGFR-1 is upregulated with respect to melanocytes. However, the expression of this receptor, in terms of percentage of positive cell lines and transcript levels, is significantly lower as compared with cell lines derived from primary lesions or lymph node metastases. In addition, in cutaneous metastases the sVEGFR$1 /$ mVEGFR-1 transcript ratio is decreased with respect to that observed in primary melanomas.

sVEGFR-1 is mainly considered a negative modulator of VEGF-A and PlGF binding to VEGFR-2 and/or mVEGFR-1. ${ }^{11}$ Consequently, high expression of the soluble receptor during the early steps of development of a primary melanoma may hamper the ability of VEGF-A and/or PlGF to sustain tumour growth, that would depend on other factors produced by tumour cells (e.g. basic fibroblast growth factor, plateletderived growth factor, interleukin-8). ${ }^{3,18}$ In agreement with 


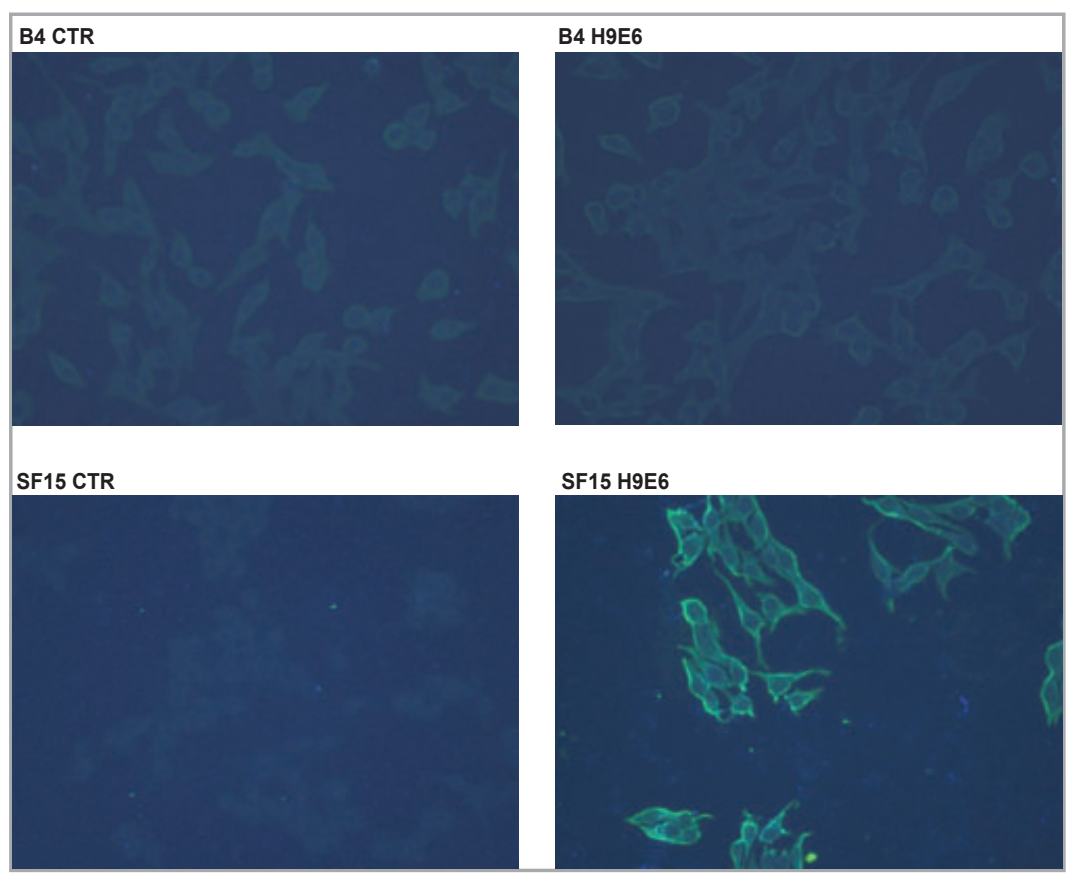

Fig 4. Specificity of the anti-soluble vascular endothelial growth factor receptor-1 (sVEGFR-1) monoclonal antibody (mAb) H9E6. Immunofluorescence staining of M14C2/SF15 cells (SF15), that express sVEGFR-1, and of M14C2/B4 cells (B4), that do not express this polypeptide, was utilized to analyse the specificity of mAb H9E6. Cells growing exponentially in Lab-tek chamber slides (Nalge Nunc Int., Naperville, IL, U.S.A.) were fixed in 2\% formaldehyde/phosphate-buffered saline (PBS) and permeabilized with $0.5 \%$ Triton X-100/PBS. Nonspecific staining was blocked overnight with $3 \%$ bovine serum albumin (BSA)/PBS at $4{ }^{\circ} \mathrm{C}$, before incubation with $\mathrm{H} 9 \mathrm{E} 6 \mathrm{mAb}\left(10 \mu \mathrm{g} \mathrm{mL}^{-1}\right.$ in $3 \% \mathrm{BSA} / \mathrm{PBS}$ at $4{ }^{\circ} \mathrm{C}$, overnight). Afterwards, cells were incubated with goat antimouse IgM antibody, stained with fluorescein isothiocyanateconjugated rabbit antigoat IgG (both $1: 100$ in 3\% BSA/PBS, $1 \mathrm{~h}$ at room temperature) and digital images were captured under a fluorescence microscope. CTR, control (not treated with mAb H9E6). Original magnification $\times 200$.

the results obtained in the analysis of melanoma cell lines, we observed that sVEGFR-1 is expressed, although at variable levels, in more than $90 \%$ of primary melanoma specimens. Moreover, a high percentage $(71 \%)$ of the cutaneous metastases analysed showed a decrease in sVEGFR-1 expression with respect to their matched primary tumours. Progression from the primary lesion to a cutaneous metastasis might be thus favoured by the downmodulation of sVEGFR-1 expression, which would contribute to augment VEGF-A and PlGF availability. A low sVEGFR-1/VEGF-A ratio in astrocytic tumour cells has been related to a higher disease malignancy. ${ }^{19}$ In this context, we have previously demonstrated the involvement in melanoma invasiveness of a VEGF-A/VEGFR-2 autocrine loop ${ }^{7}$ that would be activated when the sVEGFR1 /VEGF-A ratio in tumour cells is low, and herein we found that VEGF-A is expressed at high levels in both cutaneous metastases and primary tumours. It can be hypothesized that downregulation of sVEGFR-1 expression might occur during the switch to a metastatic phenotype, that also requires high VEGF-A expression and downmodulation of the inhibitory VEGF-A isoforms. ${ }^{20}$

Downregulation of sVEGFR-1 expression, instead, does not appear to be required for metastatic dissemination to the lymph nodes. Other members of the VEGF family, in particular VEGF-C, VEGF-D and their specific receptor in the lymphatic endothelial cells (VEGFR-3), are known to promote tumour spread towards the lymph nodes in various cancer types, ${ }^{21}$ including melanoma. ${ }^{21,22}$ Melanoma cells expressing VEGF-C induce lymphangiogenesis and lymphatic endothelial cells produce chemokines that can attract tumour cells towards the lymphatic vessels. ${ }^{23}$ Lymph node invasion might, therefore, be independent of sVEGFR-1 levels, as this receptor does not bind VEGF-C or VEGF-D.

In addition to the role of sVEGFR-1 as a negative modulator of VEGF-A, we previously demonstrated that this polypeptide can behave as a positive modulator of angiogenesis through its interaction with the $\alpha 5 \beta 1$ integrin, supporting the adhesion and migration of endothelial cells. ${ }^{13,24}$ In the present study, we found that melanoma cells may adhere to sVEGFR-1 and that this ability depends on the activation of the VEGF-A/ VEGFR-2 signalling pathway. As a component of the ECM, the sVEGFR-1 produced by both endothelial and primary melanoma cells might, therefore, contribute to tumour progression sustaining neoplastic cell mobilization towards the vascular endothelium and vice versa, with consequent melanoma dissemination through the vascular system.

In regard to mVEGFR-1, our results indicate that, even though expressed at lower levels than in primary tumours, this receptor isoform is present in almost $70 \%$ of the cell lines derived from cutaneous metastases. A moderate level of mVEGFR-1 expression is probably sufficient to favour the metastatic process, as described for melanoma and for other 


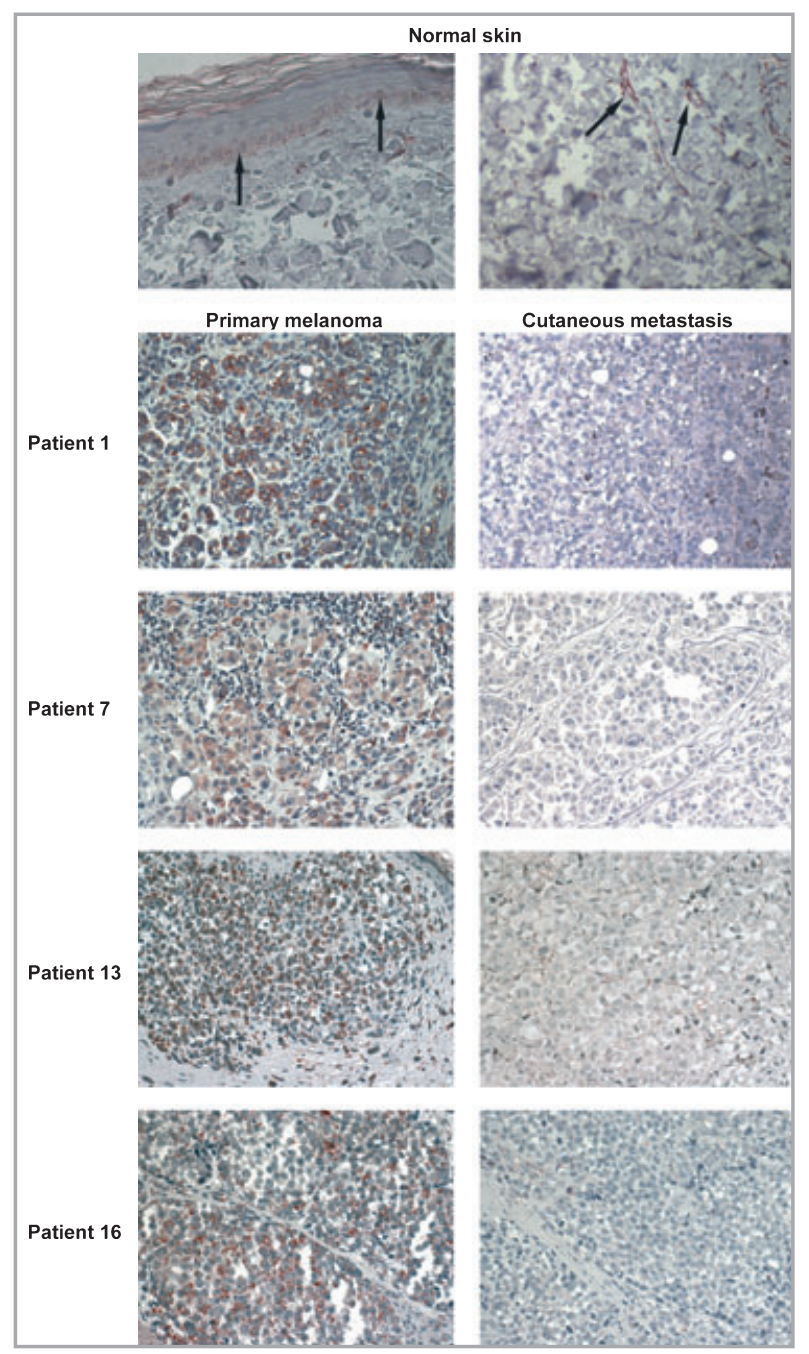

Fig 5. Soluble vascular endothelial growth factor receptor-1 expression in human melanoma specimens. Immunohistochemical staining with the monoclonal antibody (mAb) H9E6 in normal skin and in sections of four primary melanoma lesions and the cutaneous metastases from the same patients (numbered as in Table 2). The H9E6 mAb (5 $\left.\mathrm{g} \mathrm{mL}^{-1}\right)$ and a biotinylated antimouse IgM (1:300; Vector Laboratories, Burlingame, CA, U.S.A.) were used as primary and secondary antibodies, respectively. Immunoreactivity was visualized as a red cytoplasmic staining using 3-amino-9-

ethylcarbazole (Vector Laboratories) diluted in $\mathrm{H}_{2} \mathrm{O}_{2}$ as substrate, and specimens were counterstained with haematoxylin. Arrows in the normal skin panel indicate basal keratinocytes (left photograph) and vessel staining (right photograph). Original magnification $\times 200$.

kinds of neoplasia. ${ }^{25} \mathrm{~A}$ broad study on the in vivo expression of this receptor isoform in melanoma specimens has been previously published, ${ }^{26}$ and it has been proposed that this polypeptide might have a role during melanoma progression by promoting cell migration, growth and survival. ${ }^{2,6,27,28}$ Moreover, our finding of the reduction in the sVEGFR-1/mVEGFR1 ratio in cutaneous metastases indicates that variations of the balance between both receptor isoforms might contribute to the metastatic switch in melanoma.
Table 2 Expression of soluble vascular endothelial growth factor receptor-1 (sVEGFR-1) in cutaneous melanoma

\begin{tabular}{|c|c|c|c|c|}
\hline \multirow[b]{2}{*}{$\mathrm{T}$ category $^{\mathrm{a}}$} & \multirow[b]{2}{*}{ Patient } & \multirow[b]{2}{*}{$\begin{array}{l}\text { Breslow } \\
\text { thickness }^{\text {b }}\end{array}$} & \multicolumn{2}{|c|}{$\begin{array}{l}\text { sVEGFR-1 } \\
\text { immunoreactivity }^{\mathrm{c}}\end{array}$} \\
\hline & & & $\begin{array}{l}\text { Primary } \\
\text { melanoma }\end{array}$ & $\begin{array}{l}\text { Cutaneous } \\
\text { metastasis }\end{array}$ \\
\hline \multirow{3}{*}{$\mathrm{T} 1(<1 \mathrm{~mm})$} & 1 & $0 \cdot 8$ & ++ & $+/-$ \\
\hline & 2 & 0.9 & $+/-$ & NA \\
\hline & 3 & 0.95 & + & NA \\
\hline \multirow{8}{*}{$\mathrm{T} 2(1 \cdot 01-2 \mathrm{~mm})$} & 4 & $1 \cdot 22$ & ++ & NA \\
\hline & 5 & $1 \cdot 6$ & ++ & ++ \\
\hline & 6 & $1 \cdot 6$ & + & - \\
\hline & 7 & $1 \cdot 8$ & +++ & + \\
\hline & 8 & $1 \cdot 8$ & + & NA \\
\hline & 9 & $1 \cdot 8$ & ++ & $+/-$ \\
\hline & 10 & $1 \cdot 9$ & + & + \\
\hline & 11 & 2 & + & ++ \\
\hline \multirow[t]{5}{*}{ T3 $(2 \cdot 01-4 \mathrm{~mm})$} & 12 & $2 \cdot 5$ & +++ & ++ \\
\hline & 13 & 3 & +++ & + \\
\hline & 14 & 3 & +++ & ++ \\
\hline & 15 & 4 & ++ & $+/-$ \\
\hline & 16 & 4 & ++ & $+/-$ \\
\hline \multirow[t]{7}{*}{$\mathrm{T} 4(>4 \mathrm{~mm})$} & 17 & 6 & - & - \\
\hline & 18 & 6 & $+/-$ & NA \\
\hline & 19 & 7 & +++ & NA \\
\hline & 20 & 7 & +++ & NA \\
\hline & 21 & 8 & $+1-$ & - \\
\hline & 22 & $8 \cdot 5$ & ++ & NA \\
\hline & 23 & 9 & ++ & NA \\
\hline \multicolumn{5}{|c|}{$\begin{array}{l}\text { according to the American Joint Committee on Cancer mela- } \\
\text { noma staging system. }{ }^{\text {b}} \text { Tumour thickness }(\mathrm{mm}) \text {. ' } \mathrm{C} \text { Immunoreac- } \\
\text { tivity of melanoma cells: }-,<1 \% \text {; }+/-,>1 \% \text { and }<10 \% \text {; }+ \text {, } \\
>10 \% \text { and }<40 \% \text {; }++,>40 \% \text { and }<80 \% \text {; }+++,>80 \% \text {. NA, } \\
\text { not available. }\end{array}$} \\
\hline
\end{tabular}

In conclusion, our study demonstrates for the first time that sVEGFR-1 expression is upregulated in melanoma cells and suggests that this polypeptide might play a dual role during the initial phases of melanoma progression: as a soluble polypeptide that controls tumour cell invasiveness (modulating the VEGF-A/VEGFR-2 autocrine loop) and as an ECM component, directly promoting the adhesion and migration of endothelial and tumour cells. Downregulation of sVEGFR-1 in melanoma cells might increase the availability of free VEGF-A molecules capable of interacting with its membrane receptors. Thus, a low sVEGFR-1/VEGF-A and sVEGFR$1 /$ mVEGFR-1 ratio might represent an indicator of poor outcome in patients with melanoma. A better understanding of the mechanisms that regulate melanoma progression might help in the design of novel and more effective strategies for the treatment of the metastatic disease, that presently has few therapeutic options. In this context, our data suggest that the efficacy as therapeutic agents in melanoma of anti-VEGF-A mAbs might be enhanced by disrupting the interaction of sVEGFR-1 with the integrin $\alpha 5 \beta 1$. The advantage of targeting sVEGFR-1/ $\alpha 5 \beta 1$ integrin interaction will be two-fold: to 
Table 3 Adhesion of melanoma cell lines to soluble vascular endothelial growth factor (VEGF) receptor (VEGFR)-1 (sVEGFR-1)

\begin{tabular}{|c|c|c|c|c|}
\hline $\begin{array}{l}\text { Melanoma } \\
\text { cell line }\end{array}$ & $\begin{array}{l}\text { VEGF-A }^{\mathrm{a}} \\
\text { (pg per } 10^{6} \text { cells) }\end{array}$ & $\begin{array}{l}\text { VEGFR-2 }{ }^{\mathrm{b}} \\
\text { (mRNA expression) }\end{array}$ & $\begin{array}{l}\text { sVEGFR- }{ }^{\mathrm{b}} \\
\text { (mRNA expression) }\end{array}$ & $\begin{array}{l}\text { sVEGFR-1 } \\
\text { adhesion }\left(\mathrm{A}_{540}\right)^{\mathrm{c}}\end{array}$ \\
\hline ST-Mel & $1842 \pm 276$ & $1070 \pm 162$ & $17 \pm 1$ & $0.833 \pm 0.271$ \\
\hline GR-Mel & ND & $315 \pm 77$ & $568 \pm 146$ & ND \\
\hline WM115 & ND & $2293 \pm 275$ & $581 \pm 144$ & ND \\
\hline WM266-4 & $5743 \pm 599$ & $56 \pm 7$ & $19 \pm 3$ & $0 \cdot 120 \pm 0.019$ \\
\hline DR-Mel & ND & $47 \pm 2$ & $558 \pm 138$ & ND \\
\hline SK-Mel-28 & $598 \pm 72$ & $22 \pm 5$ & $149 \pm 35$ & $0.225 \pm 0.017$ \\
\hline CR-Mel & ND & $167 \pm 37$ & $1306 \pm 159$ & ND \\
\hline 13443-Mel & $792 \pm 116$ & $838 \pm 103$ & $6 \pm 1$ & $0 \cdot 185 \pm 0.010$ \\
\hline M14 & $635 \pm 56$ & 1 & 1 & ND \\
\hline
\end{tabular}

${ }^{a}$ VEGF-A secretion by melanoma cell lines was evaluated by enzyme-linked immunosorbent assay, as previously described. ${ }^{2}{ }^{b}$ VEGFR-2 and sVEGFR-1 mRNA expression in human melanoma cell lines was evaluated by quantitative real-time reverse transcription-polymerase chain reaction as described in the legend to Figure 2. 'Adhesion of melanoma cells to sVEGFR-1-coated plates after subtraction of background values (nonspecific adhesion to bovine serum albumin-coated wells) was analysed as described in the legend to Figure 6 and expressed as absorbance at $540 \mathrm{~nm}\left(\mathrm{~A}_{540}\right)$. For values $<0 \cdot 1$, adhesion was considered as not detectable (ND). All values represent the mean of at least three independent determinations $\pm \mathrm{SD}$.

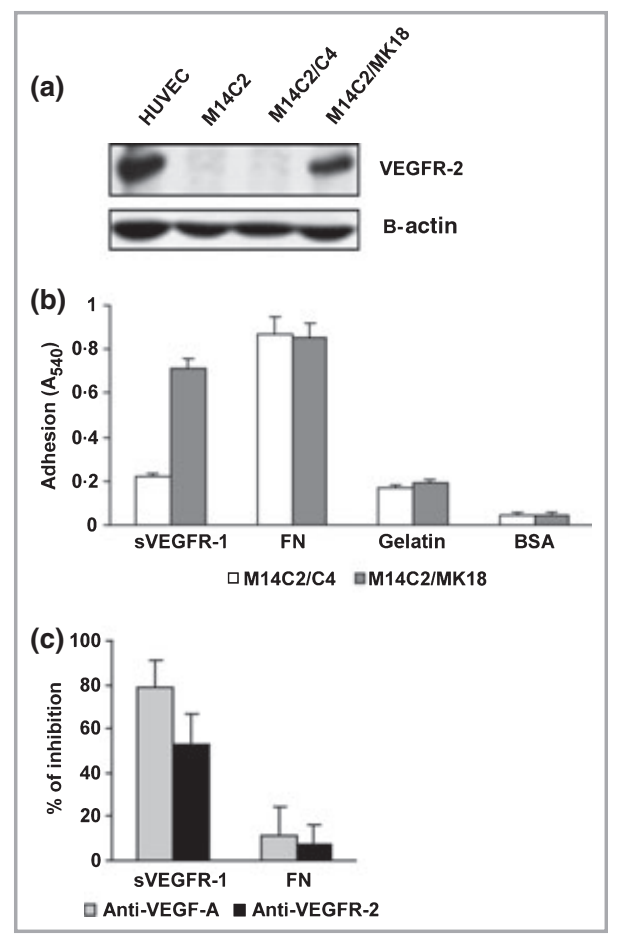

Fig 6. Adhesion of $\mathrm{M} 14 \mathrm{C} 2 / \mathrm{C} 4$ and $\mathrm{M} 14 \mathrm{C} 2 / \mathrm{MK} 18$ cells to soluble vascular endothelial growth factor (VEGF) receptor (VEGFR)-1 (sVEGFR-1). (a) Analysis of VEGFR-2 expression in M14C2/MK1 8 and control cells by Western blotting. HUVEC, human umbilical vein endothelial cells. (b) Melanoma cell adhesion was evaluated using immunological 96-multiwell plates (Becton Dickinson, Franklin Lakes, NJ, U.S.A.) coated with fibronectin (FN; Sigma, St Louis, MO, U.S.A.), chimeric VEGFR-1/Fc protein (sVEGFR-1; R\&D Systems, Abingdon, U.K.) or gelatin (Roche, Mannheim, Germany), as previously described. ${ }^{13}$ Bovine serum albumin (BSA) was used as a control for nonspecific adhesion. (c) Inhibition of cell adhesion to sVEGFR-1 and FN in the presence of $1 \mu \mathrm{g} \mathrm{mL}^{-1}$ neutralizing goat polyclonal antibodies to VEGF-A (AF-293-NA) or VEGFR-2 (AF357) from R\&D Systems. Each value represents the mean $\pm \mathrm{SD}$ of three independent experiments. abrogate the function of sVEGFR-1 as adhesion molecule and to increase the availability of SVEGFR-1 as decoy receptor capable of sequestering VEGF-A.

\section{What's already known about this topic?}

- The soluble form of vascular endothelial growth factor (VEGF) receptor (VEGFR)-1 (sVEGFR-1) behaves both as a decoy receptor, sequestering VEGF-A and placenta growth factor, and as an extracellular matrix (ECM) molecule, promoting endothelial cell adhesion and migration, but no data are available on the expression and possible role of this receptor isoform in melanoma progression.

\section{What does this study add?}

- It shows for the first time that sVEGFR-1 expression is upregulated in melanoma cells, with respect to normal melanocytes.

- It proposes a role for sVEGFR-1 during melanoma progression: as a soluble polypeptide, modulating the VEGF-A/VEGFR-2 autocrine loop, and as an ECM component, promoting tumour cell adhesion and migration.

- It suggests that low sVEGFR-1/VEGF-A and sVEGFR1/transmembrane VEGFR-1 ratios might predict a poor outcome in patients with melanoma.

\section{Acknowledgments}

The authors thank Barbara Mancini for her technical assistance in the preparation of specimens for immunohistochemical studies and Daniele Bartoloni for the artwork. 


\section{References}

1 Graeven U, Fiedler W, Karpinski S. Melanoma-associated expression of vascular endothelial growth factor and its receptors Flt-1 and KDR. J Cancer Res Clin Oncol 1999; 125:621-9.

2 Lacal P, Failla CM, Pagani E et al. Human melanoma cells secrete and respond to placenta growth factor and vascular endothelial growth factor. J Invest Dermatol 2000; 115:1000-7.

3 Mahabeleshwar GH, Byzova TV. Angiogenesis in melanoma. Semin Oncol 2007; 34:555-65.

4 Einspahr JG, Thomas TL, Saboda K et al. Expression of vascular endothelial growth factor in early cutaneous melanocytic lesion progression. Cancer 2007; 110:2519-27.

5 Marcoval J, Moreno A, Graells J et al. Angiogenesis and malignant melanoma. Angiogenesis is related to the development of vertical (tumorigenic) growth phase. J Cutan Pathol 1997; 24:212-18.

6 Graells J, Vinyals A, Figueras A et al. Overproduction of VEGF concomitantly expressed with its receptors promotes growth and survival of melanoma cells through MAPK and PI3K signaling. J Invest Dermatol 2004; 123:1151-61.

7 Lacal PM, Ruffini F, Pagani E, D’Atri S. An autocrine loop directed by the vascular endothelial growth factor promotes invasiveness of human melanoma cells. Int J Oncol 2005; 27:1625-32.

8 Marcellin M, De Luca N, Riccioni $\mathrm{T}$ et al. Increased melanoma growth and metastasis spreading in mice overexpressing placenta growth factor. Am J Pathol 2006; 169:643-54.

9 Lohela M, Bry M, Tammela T et al. VEGFs and receptors involved in angiogenesis versus lymphangiogenesis. Curr Opin Cell Biol 2009; 21:154-65.

10 Kendall RL, Thomas KA. Inhibition of vascular endothelial cell growth factor activity by an endogenously encoded soluble receptor. Proc Natl Acad Sci USA 1993; 90:10705-9.

11 Kendall RL, Wang G, Thomas KA. Identification of a natural soluble form of the vascular endothelial growth factor receptor, FLT-1, and its heterodimerization with KDR. Biochem Biophys Res Commun 1996; 226:324-8.

12 Kearney J, Kappas N, Ellerstrom C et al. The VEGF receptor flt-1 (VEGFR-1) is a positive modulator of vascular sprout formation and branching morphogenesis. Blood 2004; 103:4527-35.

13 Orecchia A, Lacal PM, Schietroma C et al. Vascular endothelial growth factor receptor- 1 is deposited in the extracellular matrix by endothelial cells and is a ligand for the $\alpha 5 \beta 1$ integrin. J Cell Sci 2003; 116:3479-89.

14 Kimura M, Kato Y, Sano D et al. Soluble form of ephrinB2 inhibits xenograft growth of squamous cell carcinoma of the head and neck. Int J Oncol 2009; 34:321-7.
15 Tentori L, Vergati M, Muzi A et al. Generation of an immortalized human endothelial cell line as a model of neovascular proliferating endothelial cells to assess chemosensitivity to anticancer drugs. Int J Oncol 2005; 27:525-35.

16 Lichtenberger BM, Tan PK, Niederleithner $\mathrm{H}$ et al. Autocrine VEGF signaling synergizes with EGFR in tumor cells to promote epithelial cancer development. Cell 2010; 140:268-79.

17 Wilgus TA, Matthies AM, Radek KA et al. Novel function for vascular endothelial growth factor receptor-1 on epidermal keratinocytes. Am J Pathol 2005; 167:1257-66.

18 Streit M, Detmar M. Angiogenesis, lymphangiogenesis, and melanoma metastasis. Oncogene 2003; 22:3172-9.

19 Lamszus K, Ulbricht U, Matschke J et al. Levels of soluble vascular endothelial growth factor (VEGF) receptor 1 in astrocytic tumors and its relation to malignancy, vascularity, and VEGF-A. Clin Cancer Res 2003; 9:1399-405.

20 Pritchard-Jones RO, Dunn DBA, Qiu Y et al. Expression of $\operatorname{VEGF}(\mathrm{xxx}) \mathrm{b}$, the inhibitory isoforms of VEGF, in malignant melanoma. Br J Cancer 2007; 97:223-30.

21 Liu B, Ma J, Wang X et al. Lymphangiogenesis and its relationship with lymphatic metastasis and prognosis in malignant melanoma. Anat Rec 2008; 291:1227-35.

22 Schietroma C, Cianfarani F, Lacal P et al. Vascular endothelial growth factor-C expression correlates with lymph node localization of human melanoma metastases. Cancer 2003; 98:789-97.

23 Achen MG, Stacker SA. Molecular control of lymphatic metastasis. Ann NY Acad Sci 2008; 1131:225-34.

24 Soro S, Orecchia A, Morbidelli L et al. A proangiogenic peptide derived from vascular endothelial growth factor receptor-1 acts through $\alpha 5 \beta 1$ integrin. Blood 2008; 111:3479-88.

25 Schwartz JD, Rowinsky EK, Youssoufian H et al. Vascular endothelial growth factor receptor-1 in human cancer: concise review and rationale for development of IMC-18F1 (human antibody targeting vascular endothelial growth factor receptor-1). Cancer 2010; 116:1027-32.

26 Mehnert JM, McCarthy MM, Aziz SA et al. VEGF, VEGFR1, and VEGFR2 expression in melanoma. J Clin Oncol 2007; 25:8520.

27 Graeven U, Rodeck U, Karpinski S et al. Expression patterns of placenta growth factor in human melanocytic cell lines. J Invest Dermatol 2000; 115:118-23.

$28 \mathrm{Wu} \mathrm{Y}$, Zhu Z. Vascular endothelial growth factor receptor 1, a therapeutic target in cancer, inflammation and other disorders. Curr Med Chem 2009; 16:2890-8. 